\title{
How can gynaecologists cope with the silent killer - osteoporosis?
}

\author{
Marian Szamatowicz \\ Medical Institute, Łomża State University of Applied Sciences, Łomża, Poland \\ Department of Reproduction and Gynaecological Endocrinology, Medical University of Bialystok, Poland
}

\begin{abstract}
Osteoporosis is a very common disease among women. It is frequently called a silent epidemic and, due to its impact on osteoporotic fractures with high morbidity and mortality, also a silent killer. There are a number of significant risk factors for osteoporosis, some of them very strongly related to the functioning of the reproductive system. These include menstrual irregularities, premature ovarian failure, early natural or surgical menopause, a high number of pregnancies, and long-term breast-feeding. Hence, there is every reason to include gynaecologists in the multidisciplinary team striving to cope with this dreadful disease. Calculation of the 10-year fracture risk, done by means of the FRAX calculator, and classification of women according to the level of risk could prove to be an effective method of limiting the negative effects of osteoporosis.
\end{abstract}

Key words: osteoporosis, risk factors, FRAX calculator, gynaecologists.

\section{Introduction}

The dramatic increase in average life expectancy is widely regarded as one of the greatest achievements of the $20^{\text {th }}$ century. Until fairly recently, people could hardly exceed the age of 50 years. Nowadays in Japan, the current leader, people live to be on average over 83 years old, and in several other countries up to at least 81 years [1]. While the population is getting older, the silent killer, the silent thief, or the silent epidemic, as osteoporosis is frequently called, has become one of the major public health problems [2]. The disease used to be characterised by low bone mass and microarchitectural deterioration of the bone tissue, leading to enhanced bone fragility and, consequently, to an increase in fracture risk. This description has been recently modified, however, and osteoporosis is now portrayed as a skeletal disorder characterised by compromised bone strength, predisposing a person to an increased risk of fracture [3]. There is abundant evidence showing that the prevalence of osteoporosis increases with age and that the age-related bone loss is greater in women than in men. Hormonal disturbances during the reproductive age and changes occurring at menopause constitute some of the major factors leading to osteoporosis in women. Ovarian ageing results in a rapid and considerable decrease in 17ß-estradiol secretion and, consequently, an increased secretion of cytokines, which activate osteoclasts. RANKL, interleukin-1 $\beta$, interleukin-6, and tumour necrosis factor are molecules that cause an increase in bone resorption, which leads to bone loss and microarchitectural deterioration, as previously mentioned [5-7]. The changes in bones constitute a serious public health problem through their close connection with age-related fractures. Lifetime risk of any fracture is very high and, according to the data presented in the report from the year 2015 in Poland, it is estimated to stand at 40\% for women and 13\% for men. In the year 2010 in Poland, there were 2,247,000 osteoporotic fractures in women and 463,000 in men. Several osteoporotic fractures, especially hip fracture, have a very high morbidity and mortality [8]. The same report reveals that the cost of treatment of osteoporosis and osteoporotic fractures is very high, amounting to nearly 3 billion PLN. Therefore, it is of great significance to recognise both the risk factors and prophylactic methods to stop the disease.

\section{Risk factors for osteoporosis}

There are many factors that can contribute to a greater likelihood of the development of osteoporosis, including age, sex, race, lifestyle, as well as certain medical conditions and treatments. Some of these are unfortunately out of our control, including:

- sex: women are much more likely to develop osteoporosis than men,

- age: the risk of osteoporosis is higher in older subjects,

- race: white women are at the greatest risk of osteoporosis, 
- family history: parents or siblings with osteoporosis increase one's risk of developing the disease,

- body frame size: people with small body frames tend to have a higher risk of osteoporosis.

Also the choice of lifestyle can influence the risk of osteoporosis. The most prevalent risk factors in this respect are as follows:

- excessive alcohol consumption: regular consumption of more than two drinks a day,

- tobacco use: smoking has been shown to diminish bone endurance.

Also of great importance are some endocrine factors in people who have increased or diminished levels of certain hormones in their bodies:

- oestrogen levels: they are considered to be some of the strongest risk factors for osteoporosis in women at menopause and those treated for breast cancer,

- thyroid diseases: the risk is greater in subjects with high concentrations of thyroid gland hormones,

- other glands: overactive parathyroid and adrenal glands increase the risk of osteoporosis.

Other risk factors, which must not be overlooked, include dietary factors, such as low calcium intake, eating disorders or gastrointestinal surgeries, administration of steroids (prevalently corticosteroids) and other medications, as well as numerous medical conditions, such as celiac disease, inflammatory bowel disease, kidney or liver disease, cancer, lupus, multiple myeloma, and rheumatoid arthritis [9, 10].

In clinical practice, the prevention of osteoporosis ought to consist of eliminating or limiting the specific recognised risk factors.

\section{Diagnosis of osteoporosis}

The proper diagnosis of osteoporosis should be considered an essential step as it offers guidance for the prevention of bone loss and fragility fractures. It should comprise both instrumental evaluation and biochemical tests. The instrumental evaluation of bone mineral measurements must provide reliable data, and for this purpose dual energy X-ray absorptiometry (DEXA) has become the most widely used technique. It facilitates the assessment of BMD (the amount of bone mass $\mathrm{g} / \mathrm{cm}^{2}$ ) of the whole skeleton or its specific sites. DEXA is usually performed at the level of lumbar spine and proximal femur $[11,12]$. The BMD test measures bone mineral density and compares it to that of an established norm or standard to give a score. $T$-score is the result of the comparison of the BMD test with an ideal or peak bone mineral density of a healthy 30 -year-old adult. A $T$-score of 0 means that the investigated BMD is equal to the norm for a healthy young adult. Differences between the investigated BMD and that of the healthy young adult norm are presented in units called standard deviations (SDs). The more SDs below 0, the lower the investigated
BMD and the higher the risk of fracture. A $T$-score between +1 and -1 is considered normal, between -1 and -2.5 as indicative of low bone mass diagnosed as osteopaenia, whereas of -2.5 or lower is indicative of osteoporosis. The greater the negative number of the $T$-score, the more severe the osteoporosis. The measurement of bone mineral density (BMD) in the assessment of osteoporosis is the most widely used parameter, but bone strength is also influenced by bone quality and tissue properties, and these are currently evaluated by means of some other available radiological techniques [13]. Nevertheless, DEXA is still a more frequently selected technique than quantitative computerised tomography (QCT) thanks to the lower cost of its use, lower radiation dose, as well as higher accuracy and shorter image acquisition time [12].

The International Osteoporosis Foundation recommends the use of a marker of bone formation (serum procollagen type I N propeptide, S-PINP) and a marker of bone resorption (serum c-terminal telopeptide of type I collagen, s-CTX) in clinical practice as markers of bone turnover for the prediction of fracture risk $[14,15]$.

\section{The assessment of fracture risk}

Calculation of the 10-year major osteoporotic fracture risk and a subsequent analysis of cost-effective treatment options allow clinicians to identify individuals who really need specific medication and to screen out those who do not. It has been recently shown that $55-70 \%$ of fractures occur in people who do not fulfil the densitometric criteria of osteoporosis. Results of numerous observations have been taken into account in order to specify clinical risk factors that can be used with or without BMD to identify people with the risk of fracture. A group of WHO experts has published a report distinguishing the following risk factors: previous fracture, parent fractured hip, smoking, glucocorticoids, rheumatoid arthritis, secondary osteoporosis, alcohol consumption exceeding three units per day, and a femoral neck BMD $T$ score of -2.5 or less. Finally, they have established an algorithm to function as a FRAX calculator (WHO Fracture Risk Assessment Tool), combining the influence of clinical risk factors of fractures with and without BMD $[16,17]$; it is available on-line at www.shef.ac.uk/FRAX. Unfortunately, there is no model designed specifically for Poland, and thus the results always refer to the English population. However, bearing in mind the reality of Polish medical care and the fact that the use of the online FRAX calculator is time-consuming, a team from Cracow has invented a modification of it called the hand-held FRAX calculator [18]. This device calculates the 10-year fracture risk and helps to assign patients to one of three groups: group $1-$ patients with a high risk of fracture, who need immediate treatment; group 2 - intermediate group - patients who need densitometric evaluation if FRAX score was 
calculated on the basis of BMI; and group 3 - low-risk group of patients who need neither treatment nor further diagnostics. The FRAX calculator is definitely not the best tool for evaluation of the fracture risk, but its simplicity makes it currently the most popular option [19]. The hand-held FRAX calculator might be a very valuable tool in the hands of medical practitioners for protecting women against osteoporosis [20, 21]. In Poland, it is now commercially available at a low cost.

\section{What can gynaecologists do in the fight against osteoporosis?}

For many women it is not an easy task to find a doctor who specialises in osteoporosis because there is no physician speciality dedicated solely to osteoporosis. As a result, women who suffer from the disease are treated by a range of medical specialists, such as endocrinologists, who treat the endocrine system, which controls the body's metabolic activity, rheumatologists, who treat and diagnose the diseases of bones, joints, muscles, and tendons, orthopaedic surgeons, who deal with the consequences of osteoporosis, geriatricians, trained on the ageing process and the conditions that often affect the elderly, family doctors, knowledgeable in many medical disciplines, internists trained in general internal medicine, and finally gynaecologists, who diagnose and treat conditions of the female reproductive system associated with osteoporosis.

The role of sex hormones in bone breakdown is very well-documented. They control the development and activity of both osteoclasts (bone breakers) and osteoblasts (bone builders). Women experience a rapid decline in bone density after menopause, when the ovaries stop producing oestrogens, especially oestradiol, the most potent form of oestrogen. The hormonal situation in perimenopausal women has been frequently described, and there is no point in repeating it here. On the other hand, there are many events in the reproductive life of women that definitely have to be recognised as risk factors of osteoporosis. This is where gynaecologists ought to do their best to limit the progression of the silent killer [22].

Irregular periods in young women may be a warning sign of hormonal disturbances that could lead to osteoporosis. Loss of menstrual regularity, secondary amenorrhoea or oligomenorrhoea constitute evidence of ovarian insufficiency and, consequently, oestrogen deficiency, which is a known risk factor for osteoporosis. The evaluation and treatment of ovarian insufficiency is a task for gynaecologists if the development of osteoporosis is to be limited in later years [23].

Primary ovarian insufficiency is not frequent but is an important cause of ovarian hormone deficiency associated with multiple health risks, including decreased bone density, and an increased risk of fractures.
Tailored hormonal replacement therapy to ameliorate bothersome menopausal symptoms can also diminish other health risks [24].

Number of pregnancies and the relationship between parity and bone mineral density. There are data that suggest that the number of pregnancies has a negative effect on BMD values and that this situation may lead to osteoporosis and fractures in the future [25].

Prolonged breast-feeding affects bone metabolism and calcium homeostasis. It can also influence the development of postmenopausal osteoporosis in highly susceptible populations [26].

Hormone replacement therapy, especially with oestrogens, has an essential impact on female bone health as it promotes the activity of osteoblasts, cells that produce bone. When considering the effect of HRT on other important disease outcomes in the global model (data of Women's Health Initiative, WHI), this kind of therapy was not recommended even in cases of high fracture risk [27]. At present, the recommendations are different. After ten years, scientists found answers to many questions and confirmed hormonal therapy (HT) (currently suggested name - menopausal hormone therapy) to be safe and effective for most newly menopausal women [28]. Recent data support the initiation of MHT around the time of menopause to treat menopause-related symptoms and to prevent osteoporosis in women at high risk of fracture $[29,30]$. Moreover, reports on some new aspects of postmenopausal osteoporosis treatment through the application of transcutaneous oestrogens supplemented with intravaginal lutein have been recently published [31].

Hormonal contraception presents different strategies. Preparations containing oestrogens have a positive effect on bones. Long-term premenopausal oral contraceptive users enter menopause with a bone density that is $2-3 \%$ higher than in non-users. Preparations containing progestogen alone have an adverse effect on BMD, causing significant bone loss $[32,33]$.

In the prevention of osteoporosis there are three very important areas that require special attention, i.e. to provide sufficient calcium and vitamin D support, to advise patients to quit smoking and reduce alcohol consumption, and to do regular exercise.

The above-mentioned risk factors for osteoporosis, a disease which very frequently does not manifest itself until pain appears or a fracture occurs, should be seriously kept in mind by gynaecological practitioners. The simplest recommended solution is to calculate the 10-year fracture risk by means of the FRAX calculator and to assign women to one of the three groups described in the section the assessment of fracture risk.

\section{Disclosure}

Author reports no conflict of interest. 


\section{References}

1. NIH publication no 11-7737, October 2011 - Global Health and Ageing

2. Cathleen S, Colon E, Saag KG. Osteoporotic fractures in older adults. Best Pract Res Clin Rheumatol 2006; 20: 695.

3. Kanis JA on behalf of the WHO Scientific Group. Assessment of osteoporosis at the primary health care level. Technical Report. WHO Collaborating Centre for Metabolic Bone Disease, University of Sheffield, UK 2007.

4. Szulc P, Bouxsein ML. Overview of osteoporosis: epidemiology and clinical management. International Osteoporosis Foundation.

5. Bord S, Ireland DC, Beavan SR, et al. The effects of estrogen on osteoprotegrin, RANKL, and estrogen receptor expression in human osteoblasts. Bone 2003; 32: 136-141.

6. Boyce BF, Xing L. Function of RANKL/RANK/OPG in bone modeling and remodelling. Archi Biochem Biophys 2008; 15: 139-146.

7. Yasuda H. RANKL, a necessary chance for clinical application to osteoporosis and cancer-related bone disease. World J Orthop 2013; 4: 207-217.

8. Czerwiński E, Synder M. Raport. Osteoporoza - cicha epidemia w Polsce. Kraków 2015.

9. Kanis JA, McCloskey EV. Risk factors in osteoporosis. Maturitas 1998; 30: 229-233.

10. Sambrook P, Cooper C. Osteoporosis. Lancet 2006; 368: 2010-2018.

11. Dasher LG, Newton CD, Lenchik L. Dual X-ray absorptiometry in today's clinical practice. Rad Clin North Am 2010; 48: 541-560.

12. Rossini M, Adami S, Bertoldo F, et al. Guidelines for the diagnosis, prevention and management of osteoporosis. Reumatismo 2016; 68: 1-39.

13. Bauer JS, Link TM. Advances in osteoporosis imaging. Europ J Radiol 2009; 71: 440-449.

14. Biver E, Chopin F, Coiffier G, et al. Bone turnover markers for osteoporotic status assessment? A systemic review of their diagnosis value at baseline in osteoporosis. Joint Bone Spine 2012; 79: 20-25.

15. Vasikaran S, Eastell R, Bruyère $O$, et al. Markers of bone turnover for the prediction of fracture risk and monitoring osteoporosis treatment: a need for international reference standards. Osteoporosis Int 2011; 22: 391-420.

16. Kanis JA, Oden A, Johansson $\mathrm{H}$, et al. FRAX and its application to clinical practice. Bone 2009; 44: 734-743.

17. Siris ES, Baim S, Nattiv A. Primary care use of FRAX: absolute fracture risk assessment in postmenopausal women and older men. Postgrad Med 2010; 122: 82-90.

18. Czerwiński E, Osieleniec J, Kumorek A. FRAX - nowe narzędzie w diagnostyce osteoporozy. Ortop Traum Rehab 2009; 11 (supl. 2): 72

19. Leslie WD, Majundar SR, Lix LM, et al. High fracture probability with FRAX usually indicates densitometric osteoporosis: implications for clinical practice. Osteoporos Int 2012; 23: 391-397.

20. Amarowicz J, Bolisega D, Rutkowski J, et al. Cost-effectiveness analysis of treatment of osteoporotic fractures in relation to FRAX algorithm in a sample of Polish population. Ortop Traumatol Rehabil 2015; 17: 59-69.

21. Rubin KH1, Friis-Holmberg T, Hermann AP, et al. Risk assessment tools to identify women with increased risk of osteoporotic fracture: Complexity or simplicity? A systemic review. J Bone Miner Res 2013; 28: 1701-1717.

22. Pluskiewicz W. Osteoporoza - kiedy ginekolog powinien podejrzewać chorobę, jak jej zapobiegać, jaką diagnostykę i leczenie wdrożyć. Ginekologia po Dyplomie 2016.

23. Alzubaidi NH, Chapin HL, Vanderhoof VH, et al. Meeting the needs of young women with secondary amenorrhea and spontaneous premature ovarian failure. Obstet Gynecol 2002; 99 (5 Pt 1): 720-725.

24. Sullivan SD, Sarrel PM, Nelson LM. Hormone replacement therapy in young women with primary ovarian insufficiency and early menopause. Fertil Steril 2016; 106: 1588-1599.

25. Gur A, Nas K, Cevik R, et al. Influence of number pregnancies on bone mineral density in postmenopausal women of different age groups. J Bone Miner Metab 2003; 21: 234-241.

26. Yun BH, Chon SJ, Choi JS, et al. The effect of prolonged breast-feeding on the development of postmenopausal osteoporosis in population with in-sufficient calcium intake and vitamin D level. Osteoporos Int 2016; 27: 2745-2753.

27. Cauley JA, Robbins J, Chen Z, et al. Effect of estrogen plus progestin on risk of fracture and bone mineral density/The women's health initiative randomized trial. JAMA 2003; 290: 1729-1738.
28. Gurney EP, Nachtigall MJ, Nachtigall LE, et al. The Women's Health Initiative trial and related studies: 10 years later: clinician's view. J Steroid Biochem Mol Biol 2014; 142: 4-11.

29. Schmidt P. The 2012 Hormone therapy Position Statement of the North American Menopausal Society. Menopause 2012; 19: 257-271.

30. Lobo Ra, Pickar JH, Stevenson JC, et al. Back to the future: Hormone Replacement Therapy as a part of a prevention strategy for the women at the onset of menopause. Atherosclerosis 2016; 254: 282-290.

31. von Mach-Szczypiński J, Stanosz S, Kościuszkiewicz J, et al. New aspects of postmenopausal osteoporosis treatment with micronized estradiol and progesterone Ginekol Pol 2016; 87: 739-744.

32. Corson SL. Oral contraceptives for the prevention of osteoporosis. J Reprod Med 1993; 38 (12 Suppl): 1015-1020.

33. Berenson AB, Radecki CM, Grady JJ, et al. A prospective, controlled study of the effects of hormonal contraception on bone mineral density. Obstet Gynecol 2001; 98: 576-582. 